\title{
CONVEX FUNCTIONS AND FOURIER COEFFICIENTS
}

\author{
HANN - TZONG WANG
}

\begin{abstract}
Let $f$ be a continuous function defined on the interval $(0,1)$. For $n=1,2, \ldots$ and $0<s<t<1$, denote by $a_{n}(f: s, t), b_{n}(f ; s, t)$ the $n$th Fourier coefficients of $f \mid(s, t)$. It is shown that the following statements are equivalent:

(i) $f$ is strictly convex on $(0,1)$.

(ii) $b_{n}(f ; s, t)<(2 / n \pi)[f(s)-f((s+t) / 2)]$ for all $n=1,2, \ldots$ and whenever $0<s<t<1$.

(iii) $b_{n}(f ; s, t)>(2 / n \pi)[f((s+t) / 2)-f(t)]$ for all $n=1,2, \ldots$ and whenever $0<s<t<1$.

If, in addition, $f$ is twice differentiable, then (i) and the following statement are also equivalent:

(iv) $a_{n}(f ; s, t)>0$ for all $n=1,2, \ldots$ and whenever $0<s<t<1$
\end{abstract}

1. Introduction. Starting from the basic definition of convex function, there are many ways to recognize a convex function, e.g., by properties of the derivatives, by geometric properties of the graph, by an integral representation and by an integral inequality. The well-known characterization of convexity in virtue of integral inequality is

$$
\frac{1}{2}(t-s) \cdot[f(t)+f(s)]-\int_{s}^{t} f(x) d x \geqslant 0, \quad a \leqslant s \leqslant t \leqslant b .
$$

This is known as the mean-value integral inequality. For generalizations, see Rado [3] and Hartman [2]; but our interest in this paper is just based on the simple geometric interpretation of the above integral inequality. It is our purpose to find some variants of the above integral inequality, which also possess geometric meanings. For this purpose, we offer here some characterizations of convexity in terms of Fourier coefficients, which are more intricate yet more incentive than the one above.

2. Characterization of convexity via sine function. Let $f$ be a continuous function defined on the interval $(0,1)$. For $n=1,2, \ldots$ and $0<s<t<1$ denote by $a_{n}(f ; s, t), b_{n}(f ; s, t)$ the $n$th Fourier coefficients of $f \mid(s, t)$, that is,

$$
a_{n}(f ; s, t)=2(t-s)^{-1} \int_{s}^{t} f(x) \cos \frac{2 n \pi(x-s)}{t-s} d x
$$

and

$$
b_{n}(f ; s, t)=2(t-s)^{-1} \int_{s}^{t} f(x) \sin \frac{2 n \pi(x-s)}{t-s} d x .
$$

We now begin with an easy lemma without proof.

Received by the editors June 18,1984

1980 Mathematics Subject Classification. Primary 26A51, 26A24.

Key words and phrases. Convex functions, Fourier coefficients and integral inequality.

(C1985 American Mathematical Society $0002-9939 / 85 \$ 1.00+\$ .25$ per page 
LEMMA 1. If $f$ is strictly convex, then $f\left(x_{2}\right)+f\left(x_{4}\right)<f\left(x_{1}\right)+f\left(x_{5}\right)$, where $x_{1}, x_{2}, x_{3}$, $x_{4}, x_{5} \in(0,1)$ with $x_{k+1}-x_{k}=x_{2}-x_{1}>0, k=1,2,3,4$.

The following lemma is based on a slight change of geometric observation for the mean-value integral inequality.

LEMMA 2. If $f$ is strictly convex on the interval $(0,1)$, then

$$
b_{1}(f ; s, t)<\frac{2}{\pi}\left[f(s)-f\left(\frac{s+t}{2}\right) s\right] \text { whenever } 0 \ll t<1 .
$$

Proof. Consider the line equation $L(x)$ satisfying $L(s)=f(s)$ and $L((s+t) / 2)$ $=f((s+t) / 2)$. Then,

$$
\int_{s}^{t} f(x) \sin \frac{2 \pi(x-s)}{t-s} d x<\int_{s}^{t} L(x) \sin \frac{2 \pi(x-s)}{t-s} d x .
$$

The result now follows from a direct computation. Q.E.D.

LEMMA 3. If $f$ is strictly convex on the interval $(0,1)$, then

$$
b_{n}(f ; s, t)<\frac{2}{n \pi}\left[f(s)-f\left(\frac{s+t}{2}\right)\right]
$$

for all $n=1,2, \ldots$ and $0<s<t<1$.

Proof. Let $x_{1}^{n}, x_{2}^{n}, \ldots, x_{2 n+1}^{n}$ be a finite sequence in $[s, t]$ with $x_{1}^{n}=s, x_{2 n+1}^{n}=t$ and $x_{k+1}^{n}-x_{k}^{n}=(t-s) / 2 n, k=1,2, \ldots, 2 n$. Then,

$$
\begin{aligned}
b_{n}(f ; s, t) & =\frac{1}{n} \sum_{k=1}^{n} b_{1}\left(f ; x_{2 k-1}^{n}, x_{2 k+1}^{n}\right) \\
& <\frac{2}{n \pi} \sum_{k=1}^{n}\left[f\left(x_{2 k-1}^{n}\right)-f\left(x_{2 k}^{n}\right)\right] \quad(\text { by Lemma 2) } \\
& =\frac{2}{n \pi}\left\{\left[f\left(x_{1}^{n}\right)-f\left(x_{2}^{n}\right)\right]+\sum_{k=1}^{n-1}\left[f\left(x_{2 k+1}^{n}\right)-f\left(x_{2 k+2}^{n}\right)\right]\right\} \\
& =\frac{2}{n \pi}\left\{\left[f\left(x_{1}^{n}\right)-f\left(x_{5}^{n}\right)\right]+\sum_{k=1}^{n-4}\left[f\left(x_{2 k+4}^{n}\right)-f\left(x_{2 k+5}^{n}\right)\right]\right. \\
& \left.-\sum_{k=1}^{n-2}\left[f\left(x_{2 k}^{n}\right)-f\left(x_{2 k+1}^{n}\right)-f\left(x_{2 k+3}^{n}\right)+f\left(x_{2 k+4}^{n}\right)\right]\right\} \\
& <\frac{2}{n \pi}\left\{\left[f\left(x_{1}^{n}\right)-f\left(x_{5}^{n}\right)\right]+\sum_{k=1}^{n-4}\left[f\left(x_{2 k+4}^{n}\right)-f\left(x_{2 k+5}^{n}\right)\right]\right\} \quad(\text { by Lemma } 1) \\
& \vdots \\
& <\frac{2}{n \pi}\left\{\left[f\left(x_{1}^{n}\right)-f\left(x_{3 m+2}^{n}\right)\right]+\sum_{k=1}^{n-3 m-1}\left[f\left(x_{2 k+3 m+1}^{n}\right)-f\left(x_{2 k+3 m+2}^{n}\right)\right]\right\}
\end{aligned}
$$

(by induction),

where $m=0,1,2, \ldots,[(n-1) / 3]$. 
There are only three cases to be considered when $m=[(n-1) / 3]$ :

(i) If $n-3 m-1=0$, then

$$
b_{n}(f ; s, t)<\frac{2}{n \pi}\left[f\left(x_{1}^{n}\right)-f\left(x_{3 m+2}^{n}\right)\right]=\frac{2}{n \pi}\left[f\left(x_{1}^{n}\right)-f\left(x_{n+1}^{n}\right)\right] .
$$

(ii) If $n-3 m-1=1$, then

$$
\begin{aligned}
b_{n}(f ; s, t) & <\frac{2}{n \pi}\left[f\left(x_{1}^{n}\right)-f\left(x_{3 m+2}^{n}\right)+f\left(x_{3 m+3}^{n}\right)-f\left(x_{3 m+4}^{n}\right)\right] \\
& <\frac{2}{n \pi}\left[f\left(x_{1}^{n}\right)-f\left(x_{3 m+3}^{n}\right)\right]=\frac{2}{n \pi}\left[f\left(x_{1}^{n}\right)-f\left(x_{n+1}^{n}\right)\right] .
\end{aligned}
$$

(iii) If $n-3 m-1=2$, then

$$
\begin{aligned}
b_{n}(f ; s, t)< & \frac{2}{n \pi}\left[f\left(x_{1}^{n}\right)-f\left(x_{3 m+2}^{n}\right)+f\left(x_{3 m+3}^{n}\right)\right. \\
& \left.-f\left(x_{3 m+4}^{n}\right)+f\left(x_{3 m+5}^{n}\right)-f\left(x_{3 m+6}^{n}\right)\right] \\
& <\frac{2}{n \pi}\left[f\left(x_{1}^{n}\right)-f\left(x_{3 m+4}^{n}\right)\right]=\frac{2}{n \pi}\left[f\left(x_{1}^{n}\right)-f\left(x_{n+1}^{n}\right)\right] .
\end{aligned}
$$

The result follows from the substitutions $x_{1}^{n}=s$ and $x_{n+1}^{n}=(s+t) / 2$. Q.E.D.

We now prove the converse of Lemma 2.

LEMMA 4. Let $f$ be a continuous function on the interval $(0,1)$. If

$$
b_{1}(f ; s, t)<\frac{2}{\pi}\left[f(s)-f\left(\frac{s+t}{2}\right)\right]
$$

for all $0<s<t<1$, then $f$ is strictly convex on $(0,1)$.

Proof. Assume not. Then there is an interval $\left(s^{\prime}, t^{\prime}\right)$ such that $D(x) \leqslant f(x)$ for all $x \in\left(s^{\prime}, t^{\prime}\right)$, where $D(x) \equiv a^{\prime} x+b^{\prime}$ is the line equation satisfying $D\left(s^{\prime}\right)=f\left(s^{\prime}\right)$ and $D\left(t^{\prime}\right)=f\left(t^{\prime}\right)$. Moreover, let

$$
P=\left\{L(x) \equiv a^{\prime} x+b: f(x) \leqslant L(x) \text { for all } x \in\left(s^{\prime}, t^{\prime}\right)\right\} .
$$

Now consider the line equation $L^{*}(x) \in P$ satisfying $L^{*}\left(s^{\prime}\right)=\inf _{L \in P} L\left(s^{\prime}\right)$. Then there is a number $x^{*} \in\left(s^{\prime}, t^{\prime}\right)$ such that $L^{*}\left(x^{*}\right)=f\left(x^{*}\right)$. If $x^{*}-s^{\prime} \leqslant t^{\prime}-x^{*}$, then let $s^{*}=s^{\prime}, t^{*}=2 x^{*}-s^{*}$ and $D^{*}(x) \equiv D(x)$. Otherwise, let

$$
P^{\prime}=\left\{L(x) \equiv a^{\prime} x+b: L(x) \leqslant f(x) \text { for all } x \in\left(2 x^{*}-t^{\prime}, x^{*}\right)\right\}
$$

and consider the line equation $D^{*}(x) \in P^{\prime}$ satisfying $D^{*}\left(x^{*}\right)=\sup _{L \in P^{\prime}} L\left(x^{*}\right)$. Then there is a number $s^{*} \in\left[2 x^{*}-t^{\prime}, x^{*}\right)$ such that $D^{*}\left(s^{*}\right)=f\left(s^{*}\right)$ and we thus let $t^{*}=2 x^{*}-s^{*}$.

Define a function $g$ on $\left(s^{*}, t^{*}\right)$ by

$$
g(x)= \begin{cases}D^{*}(x) & \text { if } x \in\left(s^{*}, x^{*}\right), \\ L^{*}(x) & \text { if } x \in\left[x^{*}, t^{*}\right),\end{cases}
$$

and consider the line equation $\bar{L}(x)$ satisfying $\bar{L}\left(s^{*}\right)=f\left(s^{*}\right)$ and $\bar{L}\left(x^{*}\right)=f\left(x^{*}\right)$. Then

$$
\begin{aligned}
\int_{s^{*}}^{t^{*}} & {[f(x)-\bar{L}(x)] \sin \frac{2 \pi\left(x-s^{*}\right)}{t^{*}-s^{*}} d x } \\
& \geqslant \int_{s^{*}}^{t^{*}}[g(x)-\bar{L}(x)] \sin \frac{2 \pi\left(x-s^{*}\right)}{t^{*}-s^{*}} d x=0 .
\end{aligned}
$$


This implies that $b_{1}\left(f ; s^{*}, t^{*}\right) \geqslant(2 / \pi)\left[f\left(s^{*}\right)-f\left(x^{*}\right)\right]$, a contradiction. Therefore, $f$ is strictly convex on the interval $(0,1)$. Q.E.D.

Combining Lemma 3 with Lemma 4, we have the following

THEOREM 1. A necessary and sufficient condition for a continuous function $f$ on the interval $(0,1)$ to be strictly convex is, for $n=1,2, \ldots$,

$$
b_{n}(f ; s, t)<\frac{2}{n \pi}\left[f(s)-f\left(\frac{s+t}{2}\right)\right] \text { whenever } 0<s<t<1 .
$$

By the same arguments when we consider the family of line equations $L(x)$ satisfying $L(t)=f(t)$ and $L((s+t) / 2)=f((s+t) / 2)$ for all $0<s<t<1$, we also have the following

THEOREM 2. A necessary and sufficient condition for a continuous function $f$ on the inverval $(0,1)$ to be strictly convex is, for $n=1,2, \ldots$,

$$
b_{n}(f ; s, t)>\frac{2}{n \pi}\left[f\left(\frac{s+t}{2}\right)-f(t)\right] \text { whenever } 0<s<t<1 .
$$

3. Characterization of convexity via cosine function. Originally, the characterization form $[4$, p. 8] which intrigues us is based on the well-known integral inequality

$$
\int_{0}^{2 \pi} f(x) \cos n x d x \geqslant 0 \text { for all } n=1,2, \ldots
$$

if $f(x)$ is convex on $(0,2 \pi)$. This result can be proved with the method of integration by parts. We offer here a geometric proof.

LEMMA 5. If $f$ is strictly convex on the interval $(0,1)$, then $a_{1}(f ; s, t)>0$ whenever $0<s<t<1$.

Proof. Let $x_{1}, x_{2}, x_{3}, x_{4}, x_{5}$ be a sequence in $[s, t]$ with $x_{1}=s, x_{5}=t$ and $x_{k+1}-x_{k}=(t-s) / 4, k=1,2,3,4$. Now consider the line equation $L(x)$ satisfying $L\left(x_{2}\right)=f\left(x_{2}\right)$ and $L\left(x_{4}\right)=f\left(x_{4}\right)$. Then,

$$
\int_{s}^{t} f(x) \cos \frac{2 \pi(x-s)}{t-s} d x>\int_{s}^{t} L(x) \cos \frac{2 \pi(x-s)}{t-s} d x .
$$

The result now follows from a direct computation. Q.E.D.

LEMMA 6. If $f$ is strictly convex on the interval $(0,1)$, then $a_{n}(f ; s, t)>0$ for all $n=1,2, \ldots$ and whenever $0<s<t<1$.

Proof. Let $x_{1}^{n}, x_{2}^{n}, \ldots, x_{n+1}^{n}$ be a finite sequence in $[s, t]$ with $x_{1}^{n}=s, x_{n+1}^{n}=t$ and $x_{k+1}^{n}-x_{k}^{n}=(t-s) / n, k=1,2, \ldots, n$. Then

$$
a_{n}(f ; s, t)=\frac{1}{n} \sum_{k=1}^{n} a_{1}\left(f ; x_{k}^{n}, x_{k+1}^{n}\right) \text {. }
$$

By Lemma 5, the result follows. Q.E.D.

We now prove the converse of Lemma 5 under a further assumption.

LEMMA 7. Let $f$ be a twice differentiable function on the interval $(0,1)$. If $a_{1}(f ; s, t)$ $>0$ whenever $0<s<<t<1$, then $f$ is strictly convex on $(0,1)$. 
Proof. Using the method of integration by parts, we have

$$
\int_{s}^{t} f(x) \cos \frac{\pi(x-s)}{t-s} d x=-\frac{t-s}{2 \pi} \int_{s}^{t} f^{\prime}(x) \sin \frac{2 \pi(x-s)}{t-s} d x .
$$

In virtue of this inequality we claim that $f^{\prime}$ is strictly increasing on the interval $(0,1)$. Assume not. Then there are only two cases to be considered: (i) $f^{\prime}$ is constant on some interval $\left(s^{\prime}, t^{\prime}\right)$. (ii) There exist two distinct points $x_{1}$ and $x_{2}$ such that $x_{1}<x_{2}$ and $f^{\prime}\left(x_{1}\right)>f^{\prime}\left(x_{2}\right)$. Case (i) implies that $a_{1}\left(f ; s^{\prime}, t^{\prime}\right)=0$, a contradiction. For case (ii), we see that by the Mean-Value Theorem for Derivatives, there exists $x^{*} \in$ $\left(x_{1}, x_{2}\right)$ such that

$$
f^{\prime}\left(x_{2}\right)-f^{\prime}\left(x_{1}\right)=f^{\prime \prime}\left(x^{*}\right)\left(x_{2}-x_{1}\right)<0 .
$$

This implies that $f^{\prime \prime}\left(x^{*}\right)<0$ and then there exists a number $r>0$ such that $x \in\left(x^{*}-r, x^{*}\right)$ implies $f^{\prime}\left(x^{*}\right)<f^{\prime}(x)$, and $x \in\left(x^{*}, x^{*}+r\right)$ implies $f^{\prime}(x)<$ $f^{\prime}\left(x^{*}\right)$. Now consider the line equation $L(x) \equiv f^{\prime}\left(x^{*}\right)$. Then,

$$
\int_{x^{*}-r}^{x^{*}+r} f^{\prime}(x) \sin \frac{2 \pi\left(x-x^{*}+r\right)}{2 r} d x>\int_{x^{*}-r}^{x^{*}+r} L(x) \sin \frac{2 \pi\left(x-x^{*}+r\right)}{2 r} d x=0 .
$$

By $(*)$, we see that $a_{1}\left(f ; x^{*}-r, x^{*}+r\right)<0$, a contradiction.

We conclude that $f^{\prime}$ is strictly increasing on $(0,1)$ and thus $f$ is strictly convex on the interval $(0,1)$. Q.E.D.

Combining Lemmas 6 and 7, we have the following

THEOREM 3. A necessary and sufficient condition for a twice differentiable function $f$ on the interval $(0,1)$ to be strictly convex is $a_{n}(f ; s, t)>0$ for all $n=1,2, \ldots$ and $0<s<t<1$.

4. Conclusions and open questions. Wright [5], looking for functions satisfying a certain set of inequalities, defines a function $f$ on the interval $(0,1)$ to be convex if, for each $0<x_{1} \leqslant x_{2}<1$ and $0<r<1-x_{2}$,

$$
f\left(x_{1}+r\right)-f\left(x_{1}\right) \leqslant f\left(x_{2}+r\right)-f\left(x_{2}\right) .
$$

We call this function $f$ Wright convex. It is well known that Wright convexity and midconvexity are equivalent for continuous functions (see [1]).

On the other hand, if $f$ is convex on $(0,1)$, then, by the Mean-Value Theorem for Integrals, there are $y_{1}, y_{2}, y_{3}, y_{4}$ such that $y_{k} \in\left(x_{k}, x_{k+1}\right)$ for $k=1,2,3,4$ and

$$
f\left(y_{2}\right)-f\left(y_{1}\right) \leqslant f\left(y_{4}\right)-f\left(y_{3}\right)
$$

in the notations in the proof of Lemma 5. The similarity of $(* *)$ and $(* * *)$ almost lead us to replace the assumption of twice differentiability with the assumption of continuity for the functions considered in Theorem 3. However, this situation becomes misty even with the assumption of continuous differentiability because 
there are functions, for example, the Weierstrass continuous functions, that are nowhere increasing and nowhere decreasing.

\section{REFERENCES}

1. G. H. Hardy, J. E. Littlewood and G. Pólya, Inequalities, Cambridge, 1934.

2. P. Hartman, Convex functions and mean value inequalities, Duke Math. J. 39 (1972), 351-360.

3. T. Rado, On convex functions, Trans. Amer. Math. Soc. 37 (1935), 266-285.

4. A. W. Roberts and D. E. Varberg, Convex functions, Academic Press, New York, 1973.

5. E. M. Wright, An inequality for convex functions, Amer. Math. Monthly 61 (1954), 620-622.

DePartment of Mathematics, University of Rochester, Rochester, New York 14627 